\title{
Gray Color Multi-Categorical Perception: Asymptotics of Psychometric Function
}

\author{
Ren Namae, Marie Watanabe, Ihor Lubashevsky \\ University of Aizu, Tsuruga, Ikki-machi, Aizu-Wakamatsu City, Fukushima 965-8560, Japan \\ E-mail for contacts: (IL) i-lubash@u-aizu.ac.jp
}

\begin{abstract}
The results of conducted experiments on categorical perception with respect to gray color categorization into three classes are reported. Namely, the subjects were instructed to categorize shades of gray generated in a random sequence into three classes: light-gray, gray, and dark-gray. The collected data are analyzed employing $(i)$ the asymptotics of the constructed psychometric functions and $(i i)$ the mean decision time in categorizing a given gray brightness. The results obtained in the reported experiments and our previous ones are compared and a plausible macro-level mechanism governing gray color categorization is discussed.
\end{abstract}

\section{Introduction}

The notion of categorical perception generally describes situations when we perceive our world in terms of discrete categories emerged previously during our communication with the social environment. Our perception is warped such that difference between objects belonging to different categories is accentuated and, in opposite, difference between objects falling into one category is deemphasized (for a review see, e.g., [1, 2]).

Previously, using virtual environment we conducted experiments on shape recognition [3] and gray color categorization [4] within the binary choice. Based on the obtained results we have put forward a hypothesis about the existence of an emergent mechanism of categorization governing our decision-making at the macrolevel rather than the level of particular neurophysiological processes. It should be noted that in spite of a vast amount of literature about various aspects of color categorization (see, e.g., [5]) the basic mechanisms governing these processes remain a challenging problem.

The pivot point of our experiments, in particular, on gray color categorization [4] is the analysis of $(i)$ the asymptotic behavior of the corresponding psychometric functions and $(i i)$ the mean decision time in classifying a given shade of gray (shade number). Figure 1 illustrates the characteristic form of the constructed psychometric functions and the mean decision time which prompted us to pose the hypothesis about the universal emergent mechanism governing the decision-making. However in these experiments we confined our investigation of categorical perception only to the binary
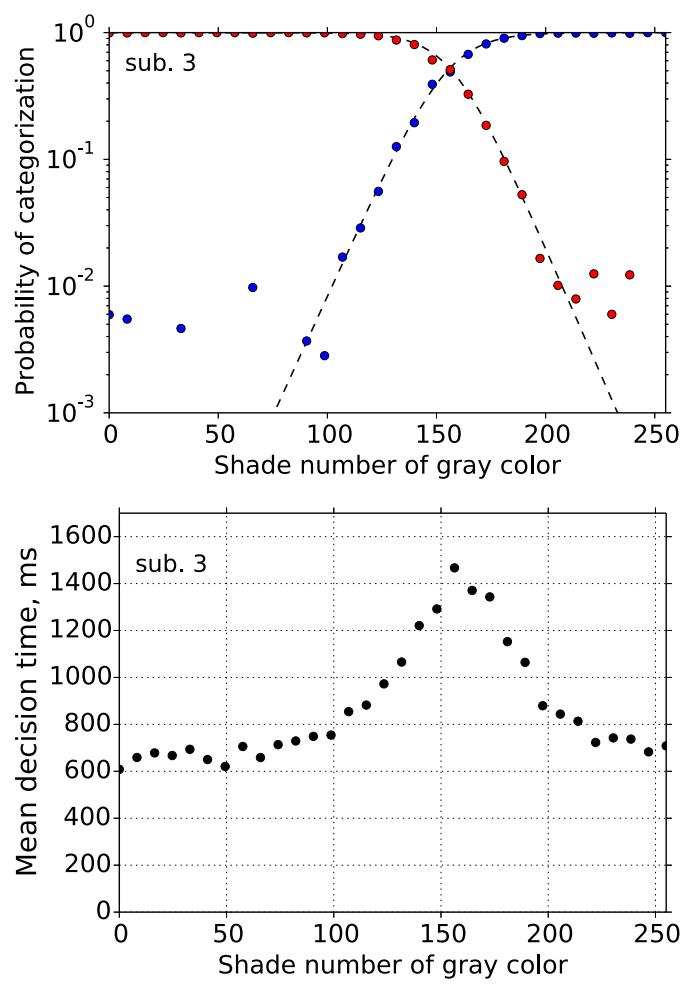

Fig. 1: Characteristic forms of the asymptotics of the corresponding psychometric functions (upper frame) and the mean decision time (lower frame) constructed based on the experiments [4] on the categorization of shades of gray withing the binary choice between lightgray (blue data points) and dark-gray (red data points). Dashed lines depicts the fitting logistic-type functions.

choice. In particular, the gray color categorization subjects were instructed to categorize shades of gray into two classes: light gray and dark gray.

The purpose of the present work is to analyze how the results obtained with the binary choice depend on the number of categorization classes and to verify the posed hypothesis about categorical perception when the number of categories excess two. In our new experiments the subjects were instructed to classify a visualized gray shade into three categories:

"ligh-gray" "gray" "dark-gray" .

These experiments were based on a simulator similar 


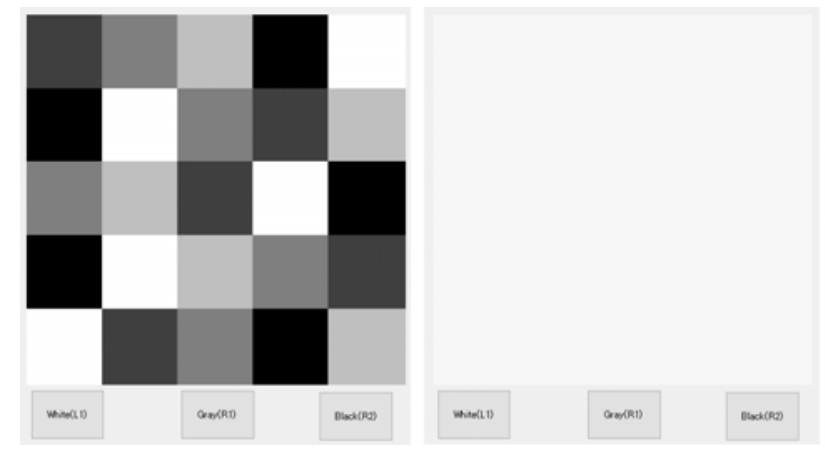

Fig. 2: Color generator

to one used in the previous experiments [4] within the same setup.

\section{Experiments}

\subsection{Color Generator}

As previously [4], in the reported experiments the following color generator was used. This generator was designed under C\# language of Microsoft Visual Studio Ultimate 2012. On Lenovo LI2221s Monitor (47.7 × $26.8 \mathrm{~cm}$ screen) a computer under the operating system Windows 10 visualizes a windows of size of $17 \times 16 \mathrm{~cm}$ with a square $\mathbb{S}$ of size of $11 \times 11 \mathrm{~cm}$ placed at it center. We divided the square $\mathbb{S}$ in twenty five parts to visualize a mosaic pattern. Color inside this square is changed during experiments; the remaining window part is filled with a neutral gray, namely, $\operatorname{RGB}(240,240,240)$. The brightness and contrast of the screen was set equal to $70 \%$ and $60 \%$, respectively. To get subject's response to a visualized color we used a standard game joystick.

Each trial of shade categorization is implemented as follows. A random integer $I \in[0,255]$ is generated uniformly and the area $\mathbb{S}$ is filled with the gray color $G(I):=R G B(I, I, I)$. Then a subject has to classify the visualized gray color $G(I)$ according to his/her perception into categories three possible categories: light gray, gray, and dark gray. A subject's choice is recorded via pressing one of three joystick buttons. Then a mosaic pattern of various shades of gray is visualized for $500 \mathrm{~ms}$. This mosaic pattern is used to depress a possible interference between color perception in successive trials that can be caused by human iconic memory. After that a new number $I$ is generated uniformly and the next trial starts.

The same computer was used for all the experiments.

\subsection{Experimental Setup}

At the first step of experiments the subjects were trained to operate with the color generate via the joystick within about fifty trials of classifying visualized shades of gray. Three joystick buttons were used for fixing a choice made in categorizing a given color, the left one matched the light-gray choice, the middle one matched the gray choice, and the right one matched the dark-gray choice. The visible labels "White", "Gray" and "Black" on the window are arranged in the same order (Fig. 2) to prompt subjects the appropriate joystick button for pressing.

The experiments were set up as follows. Three subjects were involved in this experiments. They are all male students of age 21-22 years old.

The experiments spanned over 4 days. We have recorded 2000 data point per day and the total is 8000 data point for one subject. This amount of data is crucial because, on one hand, only the asymptotic behavior of psychometric functions bears the information about possible mechanisms governing subject's decision-making. On the other hand, the relative volume of collected data related to this asymptotics is rather small.

The data records were saved in CSV-files, where each line $k$ contains three data values, namely,

- 1st column: the integer $I_{k}$ specifying the shade $G_{k}=\operatorname{RGB}\left(I_{k}\right)$ of displayed gray,

- 2nd column: the duration $\Delta_{k}$ of the time interval between displaying the color $G_{k}$ and pressing a button by subject,

- 3rd column: ID of the pressed button, $J_{k}$, equal to 0 for the "light-gray" choice, 1 for the "gray" choice and 2 for the "dark-gray" choice.

\section{Analysis}

The collected data have been used for constructing the following four functions. The first one is psychometric function for the "light-gray" category, i.e., the probability $P_{w}(I)$ of classifying a given shade $I$ as lightgray. The second and third ones, $P_{g}(I)$ and $P_{b}(I)$, respectively, are similar functions for the "gray" and "dark-gray" categories. In these experiments only three categories are used so for each value $I$ the equality

$$
P_{w}(I)+P_{g}(I)+P_{b}(I)=1
$$

holds. The last function, $T(I)$, is the mean decision time of choosing any one of these categories for a given shade of gray.

The psychometric function of choosing, e.g., the "gray" category has been constructed as follows:

$$
P_{g}(I)=\left[\sum_{k=1}^{M} \delta\left(I, I_{k}\right) \delta\left(1, J_{k}\right)\right] \cdot\left[\sum_{k=1}^{M} \delta\left(I, I_{k}\right)\right]^{-1}
$$

where $k$ is the index of recorded data point, $M=8000$ is the total number of records, and $\delta(i, j)$ is the Kronecker delta:

$$
\delta(i, j)= \begin{cases}1, & i=j \\ 0, & i \neq j\end{cases}
$$



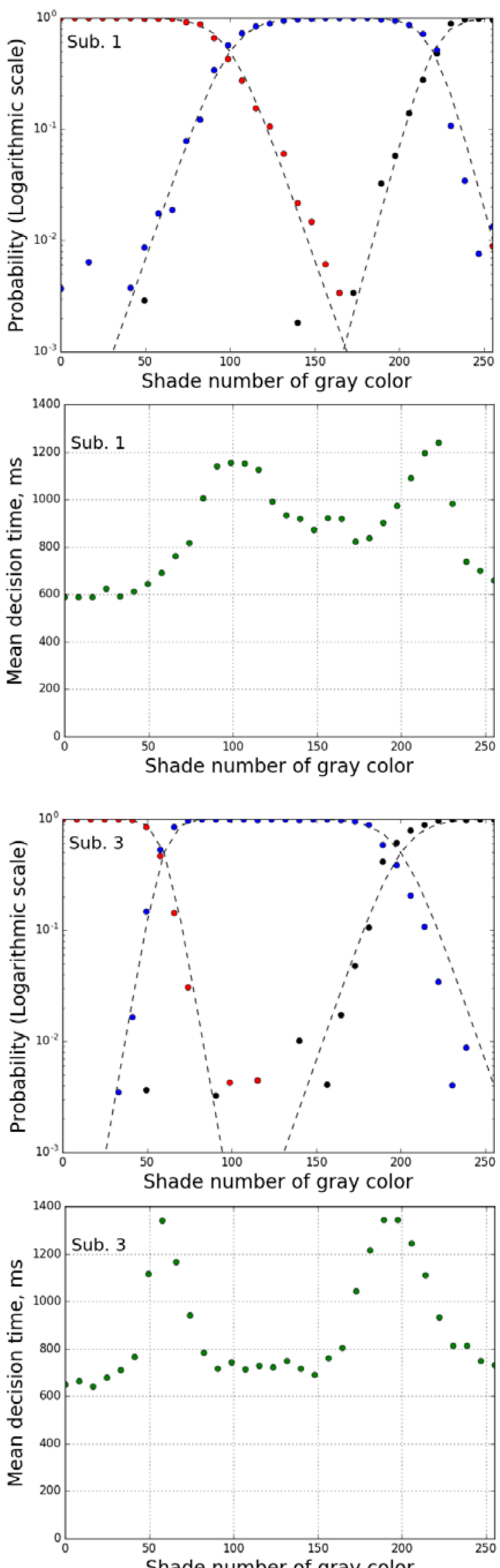

Shade number of gray color
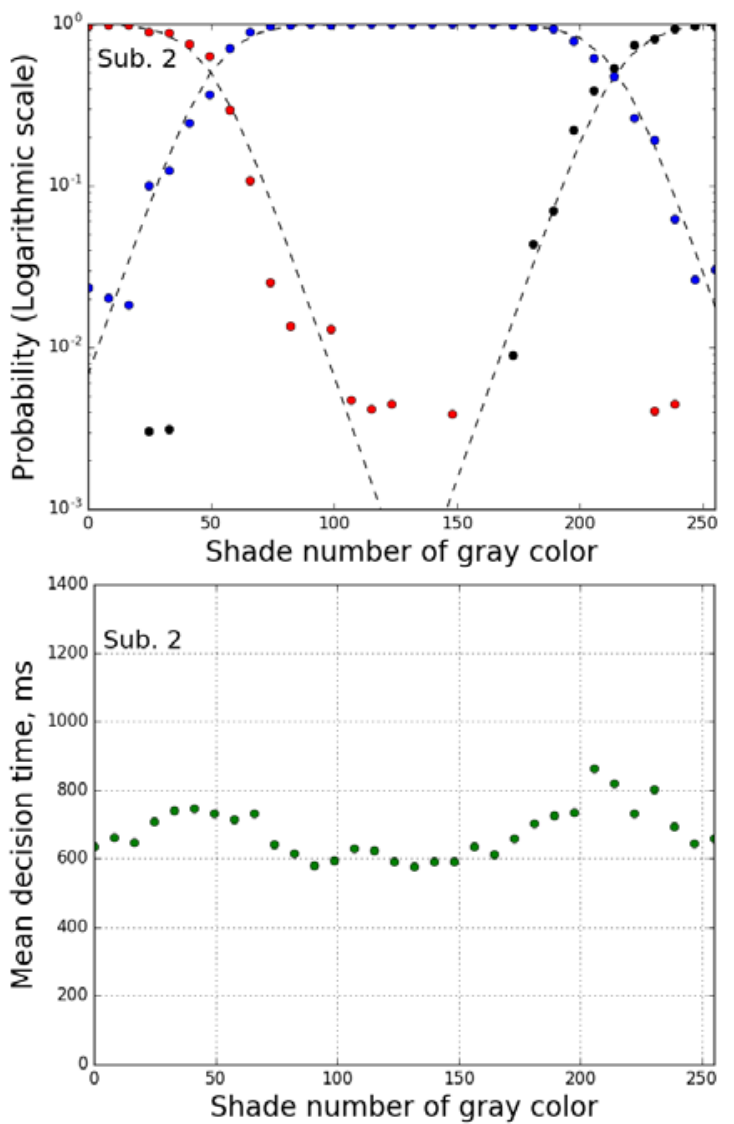

Fig. 3: Psychometric functions and the mean decision time vs the shade of gray, $\operatorname{RGB}(I, I, I)$ for three subjects involved in the reported experiments.

In the frames showing the psychometric functions in log-normal scales data points corresponding to the "light-gray," "gray," and "dark-gray" categories are shown in black, blue, and red, respectively. Dashed lines represent the fitting logistic-type functions specified by Exp. (4). The frames are arranged in such a way that the psychometric function plot and the mean decision time plot combined together for each subject individually be placed one above the other. This arrangement makes it clear that the peaks in the dependence of mean decision time on the shade index correspond to the regions where the uncertainty in gray color categorization is most pronounced. 
The function $T(I)$ - the mean decision time vs the shade of gray $(I)$ - has been constructed as follows

$$
T(I)=\left[\sum_{k=1}^{M} \delta\left(I, I_{k}\right) \Delta_{k}\right] \cdot\left[\sum_{k=1}^{M} \delta\left(I, I_{k}\right)\right]^{-1} .
$$

The obtained results are demonstrated in Fig. 3.

\section{Results and Discussion}

The constructed psychometric functions are depicted in Fig. 3 for all three subjects. As seen, there are two common properties exhibited by the psychometric functions. The first one is the exponential form of their asymptotic behavior. In the shown log-normal plots it is represented by a rather close location of data points around straight lines being the asymptotics of the fitting functions:

$$
P_{w, g, b}(I) \approx \frac{1}{2}\left\{1+\tanh \left[ \pm \frac{\left(I-I_{m: w, g, b}\right)}{\delta I_{w, g, b}}\right]\right\},
$$

where $I_{m: w, g, b}$ is the point of the crossover region and the value $\delta I_{w, g, b}$ characterizes its thickness. Naturally the specific values of the parameters $I_{m: w, g, b}$ and $\delta I_{w, g, b}$ may change from subject to subject. Similar results are obtained in our previous experiments on binary gray color categorization presented in Fig. 1.

The second common properties is that in categorizing any given shade of gray the subjects made choice only between two classes. Indeed, in the crossover regions the data points correspond to the subject's choice either between "light-gray" and "gray" or between "gray" and "dark-gray." For example, the crossover region of the choice between "light-gray" and "gray" practically does not contain the data points matching the "dark-gray" category.

These results allow us to suppose that the mechanism governing the uncertainty in subject's decision-making in categorizing the gray shades admits interpretation as the potential type mechanism. It means that the subject's decision-making is treated as a random process $\zeta$ (where $\zeta$ is the state variable of some system) in a potential field $U(\zeta, I)$ depending on the shade index $I$ as a parameter. In this case the subject's choice of a category $z_{\alpha}$ at trial $k$ for a given shade index $I$ is described by the steady-state probability of finding the system inside the potential well located at the point $z_{\alpha}$ :

$$
P_{\alpha} \approx \exp \left\{-U_{\alpha}(I)\right\}\left[\sum_{\alpha^{\prime}} \exp \left\{-U_{\alpha^{\prime}}\right\}\right]^{-1}
$$

Here

$$
U_{\alpha}(I):=\left.U(\zeta, I)\right|_{\zeta=\zeta_{\alpha}}
$$

and we have assumed all the potential wells are to be rather deep to ignore the contribution of other system states to probability (5). In the general case when for a certain value of the grade index $I$ the contributions of two potential wells become comparable, the other potential wells remain ignorable. So withing the linear approximation of the potential dependence on the parameter $I$

$$
U(\zeta, I+\delta I) \approx U(\zeta, I)+\frac{\partial U(\zeta, I)}{\partial I} \cdot \delta I
$$

expression (5) leads to formula (4). Other models like detecting noisy signals give another type asymptotics of psychometric functions.

The mean decision time $T(I)$ with its relation to the psychometric functions is also depicted in Fig. 3. These plots show how the mean time required for the subjects to make decision about classifying a current shade of gray changes with the shade number $I$. For the values of $I$ corresponding to the crossover regions the patterns $T(I)$ contain peaks. These peaks are pronounced for Subjects 1 and 3, for Subject 2 they are not sharp, nevertheless, are visible. In particular, for all the subjects the mean decision time is found to be about $600-700 \mathrm{~ms}$ outside these peaks, which can be regarded as the upper boundary $T_{p}$ of the human response delay time controlled by physiological processes of recognizing threshold events within their unpredictable appearance. At least, it is the upper boundary of visual time intervals presenting timescales relevant to natural behavior, see, e.g., [6] and references therein.

In the crossover regions the uncertainly in subject's category choice is most essential because for such values of shade number choosing one of two categories becomes equiprobable. Here the peak values $T_{m}$ of the mean decision time exceed $T_{p}$ substantially, e.g., for Subject 3 $T_{m} \approx 1.4 \mathrm{~s}$. The same property of the $T(I)$-pattern is found in our previous experiments [4] on binary categorization of gray color. It should be also noted that a similar dependence of the decision time was found in the speech recognition [7], however, the time delay attained in the peak maximum does exceed $600 \mathrm{~ms}$. In our data the mean time of decision-making includes also a time interval between making decision and pressing the corresponding button. However, if the time delay is deducted from the measured time data, the found peak will be even more pronounced.

The appearance of these peaks at the $T(I)$-patterns can be explained by turning to the concept of dual system of decision-making. This concept accepts the existence of the automatic and intentional systems contributing simultaneously to human response and being in a continuous interplay with each other. The formerautomatic system - is reflexive, fast, affective, associative, and primitive. The latter - intentional system - is deliberative, controlled, slow, cognitive, propositional, and more uniquely human. Besides, there are accounts assuming the dual-processes to arise parallel and compete with each other. However, there are also arguments against the dual system of decision-making; for a review and discussion of the evidence supporting 
both sides of debate a reader may be referred, e.g., to Refs. $[8,9]$. The found peaks of the mean decision time dependence on the shade number argue for that the two cognitive systems do exist and are comparable in their influence on the categorical perception. Indeed, the characteristic time scale of decision-making in categorical perception depends substantially on the uncertainty in the subject's choice. It argues that cognitive (mental) processes should be involved in decision-making when the choice of the appropriate color category is not evident.

\section{Conclusion}

In the present work we have reported the results of our experiments on categorical perception of shades of gray when the number of classification classes is three. Three subjects were involved in these experiments and instructed to categorize them into the classes: lightgray, gray, and dark-gray. The collected experimental data are analyzed using the asymptotics of psychometric functions and the mean decision time in choosing the appropriate color class as a function of the shade number.

The obtained results together with results of our previous experiment $[3,4]$ argue for the following hypothesis:

- Categorical perception, at least, of shades of gray, is governed by a potential mechanism of decisionmaking treated as a random process in a potential field whose profile depends on a given shade number as a parameter. Within this approach the classification classes match wells in the corresponding potential relief.

- The characteristic time scale of decision-making in categorizing the shades of gray, at least in the analyzed case, depends substantially on the uncertainty in classifying a given shade; the higher the uncertainty, the longer the decision time. The obtained data enable us to relate this effect with considerable contribution of mental processes to categorization. We regard the feature as a certain argument for the existence of the dual system of cognitive processes.

- When it is physically possible and different categories are independent, i.e., they are not mixed in the mind-which is the case in the gray color categorization-humans prefer to make choice between only two categories of classification for a given object or stimulus, whereas other categories are not taken into account. We call it the principle of pair-wise categorization.

It should be noted that the posed hypotheses are based on the cumulative results obtained in three series of experiments involving as a whole approximately ten dif- ferent subjects, which enables us to expect their reproducibility and weak dependence on human individuality.

\section{References}

[1] Robert L. Goldstone and Andrew T. Hendrickson. Categorical perception. Wiley Interdisciplinary Reviews: Cognitive Science, 1(1):69-78, 2010.

[2] Stevan Harnad. To Cognize is to Categorize: Cognition is Categorization. In Henri Cohen and Claire Lefebvre, editors, Handbook of Categorization in Cognitive Science, chapter 1, pages 20-45. Elsevier Ltd., Amsterdam, 2005.

[3] Ihor Lubashevsky and Marie Watanabe. Statistical Properties of Gray Color Categorization: Asymptotics of Psychometric Function. In Proceedings of the 47th ISCIE International Symposium on Stochastic Systems Theory and Its Applications, Honolulu, Dec. 5-8, 2015, pages 41-49, Kyoto, 2016. Institute of Systems, Control and Information Engineers (ISCIE).

[4] Minoru Kobayashi. Asymptotic Behavior of the Psychometric Function of Shape Recognition. Master's thesis, University of Aizu, 2014.

[5] Henri Cohen and Claire Lefebvre, editors. Handbook of categorization in cognitive science. Elsevier, Amsterdam, 2005.

[6] J. P. Mayo and M. A. Sommer, Neuronal correlates of visual time perception at brief timescales. Proceedings of the National Academy of Sciences, 110(4):1506-511, 2013.

[7] G. M Bidelman, S. Moreno, and C. Alain. Tracing the emergence of categorical speech perception in the human auditory system. Neuroimage, 79:201$212,2013$.

[8] J. Evans. Dual-processing accounts of reasoning, judgment, and social cognition. Annual Review of Psychology, 59:255-78, 2008.

[9] A. Rustichini. Dual or unitary system? Two alternative models of decision making. Cognitive, Affective, \&5 Behavioral Neuroscience, 8:355-362, 2008. 